\title{
Coincidencias y divergencias: arquitectura e instalaciones artística
}

COINCIDENCES AND DIVERGENCES: ARCHITECTURE AND ARTISTIC INSTALLATIONS

\author{
Gonzalo Enrique Bernal-Rivas*
}

* Universidad de Guanajuato,

México

Correo-e: artkitectonic@gmail.com Recibido: 12 de abril de 2020

Aprobado: 3 de agosto de 2020
Resumen: Se presenta un estudio comparativo cuyo objetivo es destacar las similitudes y diferencias que existen entre la arquitectura y las instalaciones artísticas. Se analizan cuatro casos de estudio, y en cada uno de ellos se contrasta un par de obras procedentes de ambas disciplinas, mismas que fueron seleccionadas considerando el uso común de ciertos recursos visuales: One-Sqm-House, de Van Bo Le-Mentzel y Measurement Rooms, de Mel Bochner; Jade Eco Park, de Philippe Rahm, Inert Gas Series y Ultrasonic Wave Piece, de Robert Barry; Roulotte, de Xavier Arenòs, Domēnec, Martí Peran y Just about Virtues and Vices in General, de Erwin Wurm; el Centro de refugiados, de Hans-Walter Müller y paraSITE homeless shelter, de Michael Rakowitz. Este análisis permite vislumbrar la gran diversidad de aspectos que aproximan o separan a estos dos mundos vinculados por su interés en el espacio.

Palabras clave: historia del arte; arte contemporáneo; creación artística; artes visuales; arquitectura; espacio

Abstract: A comparative study is presented whose objective is to highlight the similarities and differences that exist between architecture and artistic installations. Four case studies are analyzed, and in each one of them a couple of works from both disciplines are contrasted, which were selected considering the common use of certain visual resources: One-Sqm-House, by Van Bo Le-Mentzel and Measurement Rooms, by Mel Bochner; Jade Eco Park, by Philippe Rahm, Inert Gas Series and Ultrasonic Wave Piece, by Robert Barry; Roulotte, by Xavier Arenòs, Domēnec, Martí Peran and Just about Virtues and Vices in General, by Erwin Wurm; the Hans-Walter Müller Refugee Center and Michael Rakowitz's paraSITE homeless shelter. This analysis allows us to glimpse the great diversity of aspects that bring together or separate these two worlds linked by their interest in space.

Keywords: art history; contemporary art; artistic creation; visual arts; architecture; space 
E ste artículo consta de tres partes. En la primera, se revisa brevemente el origen de las instalaciones artísticas y su interés por el espacio, en el contexto del pensamiento de principios del siglo XX. También se enfatizan las décadas de 1960 y 1970 como un momento histórico especialmente importante para su desarrollo. Desde ese marco, en la segunda sección se destacan los conceptos de 'perceptibilidad' y 'portabilidad', a partir de los cuales se establecen dos parejas de dicotomías, dando como resultado cuatro casos de estudio. Cada uno de estos se analiza a partir de un par de ejemplos formados por una instalación y una obra arquitectónica, los cuales fueron seleccionados tomando como criterio la similitud en el uso de determinados recursos visuales. Finalmente, en la última parte se señalan las convergencias y divergencias encontradas a partir de los casos de estudio establecidos.

El espacio como materia de creación es un concepto central para la arquitectura desde sus orígenes más remotos. Este interés ha sido compartido por las artes visuales en el pasado reciente, particularmente, desde el surgimiento de las primeras instalaciones a principios del siglo XX. En ese momento histórico:

La extraordinaria importancia de las transformaciones [...] y el creciente protagonismo del espacio en todos los ámbitos de la vida y el pensamiento se muestran en la mayoría de los discursos teóricos de la época, en los nuevos campos científicos abiertos a partir de las combinaciones espacio/temporales de Albert Einstein, o en los estudios psicológicos, perceptivos y cognitivos derivados de la nueva 'cartografía' espacial conformada desde las condiciones históricas, físicas, discursivas e individuales del propio sujeto (Larrañaga, 2006: 9).

Dos de los ambientes (nombre que recibieron las instalaciones) más importantes de este periodo fueron las tres 'estructuras Merz'1 (la primera de elllas, construida en 1923) del alemán Kurt Schwitters (1887-1948), creadas con la intención de hacer coincidir el arte y la vida en un espacio transitable; y el restaurante L'Aubette (19271928), en Estrasburgo, hecho por el holandés Theo van Doesburg (1883-1931) y el francés Hans Arp (1886-1966) como una materialización de la idea de que no hay diferencia entre las artes. También destaca la intervención Mile of string [Milla de cuerda] (1942), del francés Marcel Duchamp (1887-1968), una telaraña de cuerdas que ocupaba el espacio que rodeaba las pinturas de una exposición surrealista presentada en Nueva York para explorar la manera en que el espacio se integraba como elemento compositivo.

Los orígenes más significativos de las instalaciones pueden encontrarse en las décadas de 1960 y 1970. El término 'ambiente' siguió usándose entonces para describir un conjunto de obras que, entre otros rasgos, estaban "comprometidas con su espacio circundante -un rechazo flagrante de la práctica artística tradicional-e incorporaban a los espectadores [...] Expansivas e incluyentes, estaban destinadas a ser catalizadores de nuevas ideas, no receptáculos de significados fijos" (Dempsey, 2010: 247). ${ }^{2}$ Dos de las obras más relevantes de ese período fueron Le Vide [El vacio] (1958), del francés Yves Klein (1928-1962), en la que el artista presentó una galería vacía; y Le Plein [Lo lleno], del francés Arman (1928-2005), en la que el creador llenó la misma galería de basura.

En estos años también se dio una importante aproximación entre el arte y la arquitectura. Destaca, por ejemplo, la influencia que la

1 “El término 'merz' usado por el artista provenía de la terminación de 'commerz' (comercio) sacada de un anuncio bancario, según el propio autor, y, a su vez, del comienzo de 'merzbau' (ambiente)" (Larrañaga, 2006: 16).

2 "engaged with their surrounding space — a flagrant rejection of traditional art practice - and incorporated viewers into the works. Expansive and encompassing, they were intended as catalysts for new ideas, not receptacles of fixed meanings" [La traducción es mía]. 
Internacional Situacionista tuvo sobre el grupo de arquitectos ingleses Archigram (1960-1974). Conviene señalar, particularmente, Nueva Babilonia (1956-1974), un proyecto utópico de ciudad nómada planteado por el situacionista holandés Constant (1920-2005), que tuvo un profundo eco sobre Archigram, en algunos de cuyos proyectos encontramos estructuras inflables, por ejemplo, The Cushicle, de Michael Webb (1964). Estos acercamientos han sido explorados en exposiciones como "Construir el aire. Arquitectura y diseño inchable, 1960-1975", presentada en el Centro Pompidou Málaga entre 2018 y 2019.

Diferentes aspectos del espacio han sido tratados a lo largo del tiempo. Dos de ellos son la percepción espacial, un tema de interés para todas las áreas del conocimiento (las artes no fueron la excepción), en especial, durante la primera mitad del siglo XX; y la portabilidad, un concepto derivado de la idea de ciudad nómada propia de la segunda mitad del siglo XX y que se ha extendido hasta nuestros días. A partir de estos atributos se pueden establecer, para desarrollar el estudio comparativo deseado, dos dicotomías. La primera, abarca obras arquitectónicas e instalaciones que han usado elementos perceptibles o no perceptibles como recurso visual. La segunda, incluye piezas de ambas disciplinas que han empleado piezas portátiles no inflables e inflables. A partir de estas dos parejas de dicotomías proponemos cuatro casos de estudio. En cada uno de ellos se analizan dos ejemplos integrados por una instalación y una obra arquitectónica con el objetivo de encontrar las convergencias y divergencias que existen. La selección de los trabajos presentados se llevó a cabo tomando como criterio la presencia de un recurso visual en común.

\section{Caso 1: el uso de elementos perceptibles}

En este primer caso, nos referimos a un recurso visual que ha sido compartido por las instalaciones y la arquitectura, consistente en acentuar un rasgo del espacio, sus dimensiones. En 2012, el arquitecto laosiano Van Bo Le-Mentzel (1977) creó el proyecto One-Sqm-House, una casa móvil de madera de $40 \mathrm{~kg}$ que ocupa solamente $1 \mathrm{~m}^{2}$. La obra está montada sobre ruedas y puede colocarse vertical u horizontalmente, dependiendo de la actividad que se quiera realizar en ella. El propio arquitecto la ha definido así:

Es un espacio diminuto si te quedas en esa casa todo el día, pero si defines a tu casa como un tipo de base central y declaras al parque como tu jardín o a la ciudad como tu sala, esta casa es probablemente la más grande que puedas imaginar (CNN, 2012). ${ }^{3}$

Van Bo Le-Mentzel siempre se preguntó qué es el hogar. Y una de las respuestas que encontró fue One-Sqm-House, gracias a la cual él puede establecerse donde desee. La idea del arquitecto es que cualquier persona pueda construir su propia casa, por lo que los planos están disponibles para quien los solicite y, a cambio, pide que los interesados compartan con él su punto de vista acerca de la ciudad.

Por otra parte, en las artes visuales, a partir de la muestra "Los dibujos activos y otras cosas visibles sobre papel no tienen por qué ser considerados necesariamente como arte", presentada en el Museo Judío en 1966 y estimada por muchos como la primera exposición completamente conceptual, el trabajo del artista estadounidense Mel Bochner (1940) fue considerado como 'pensamiento hecho visible'. Con esta intención creó, desde 1969, su serie Measurement Rooms, que consistió en exhibir las medidas de las salas de exposición vacías, fijándolas directamente con etiquetas a los diferentes elementos arquitectónicos que las conformaban.

3 "It's a tiny space if you stay in that house the whole day, but if you define your house as a kind of central base and declare the park as your garden, or the city as your living room, this house is possibly the biggest house that you can imagine" [ $\mathrm{La}$ traducción es mía]. 
One-Sqm-House y Measurement Rooms tienen en común que persiguen modificar, desde su disciplina, el pensamiento del espectador/usuario respecto al espacio, enfrentándolo a una realidad que a veces no se puede o no se quiere ver. Además, el hecho de que ambos proyectos revelen las dimensiones de los elementos que integran el espacio nos remite a pensar en conceptos como la transparencia, por la que ambos creadores optaron al hacer evidente información que normalmente permanece oculta para conseguir su objetivo; y la honestidad, en el sentido de que cada uno señala una verdad.

Estas dos obras difieren en que la pieza arquitectónica está interesada en que el usuario conceptualice el espacio exterior de una manera diferente, como una extensión del espacio interior; mientras que la instalación reflexiona sobre el hecho de que la idea que origina el espacio es tan importante como el propio espacio. Otra divergencia importante es que One-Sqm-House se refiere a las dimensiones de la casa mediante las letras presentes en el nombre del proyecto, y Measurement Rooms usa números.

\section{Caso 2: el uso de elementos imperceptibles}

El caso contrario al anterior es aquel en el que los creadores, tanto desde la arquitectura como desde las instalaciones, han tratado de hacer que algunos elementos que integran el espacio no sean notorios. Para ilustrar esta segunda tipología presentamos una obra del arquitecto suizo Philippe Rahm (1967) y otra del artista estadounidense Robert Barry (1936), que comparten el uso de elementos imperceptibles como recurso visual, específicamente la emisión de gases y de ondas radioeléctricas no audibles.

Desde la arquitectura, Rahm ya había utilizado estos recursos, aunque con un propósito diferente. Su proyecto Jade Eco Park (Rahm, 2016), un parque con una extensión de 40 ha ubicado en Taichung, Taiwán, tiene como objetivo favorecer que las personas que se encuentran en esa ciudad visiten los espacios exteriores, lo cual no resulta fácil tomando en cuenta el clima subtropical de la región. Para conseguirlo, Rahm propuso crear un espacio suficientemente cómodo para los usuarios en el que la temperatura, la humedad y la contaminación fueran controladas. La estrategia consistió en identificar las zonas más frescas, secas y limpias para aumentar su diferencia respecto a otras áreas. Cada uno de estos parámetros (temperatura, humedad y contaminación) se plasmó en un mapa, el cual se materializó usando dispositivos naturales y artificiales de tres tipos: de enfriamiento, de secado y de descontaminación. Uno de los dispositivos de descontaminación artificial se llama 'eclipse de ozono' y sopla aire filtrado que no contiene $\mathrm{NOx}, \mathrm{O}^{3}$ ni $\mathrm{SO}^{2}$. Otro artefacto similar, denominado 'corriente preindustrial', provee aire sin partículas PM10 y PM2.5, que son producidas por la industria y los coches. Además, el parque cuenta con un repelente ultrasónico que emite ondas sonoras menores a $20 \mathrm{kHz}$, imitando el movimiento de las alas de las libélulas, las cuales son los depredadores naturales de los mosquitos. Estos sonidos se encuentran en un rango inaudible para los humanos.

Por otra parte, a finales de los años sesenta, Barry estuvo interesado en abordar la desmaterialización del arte. En Inert Gas Series/Helium, Neon, Argon, Krypton, Xenon/From a Measured Volume to Indefinite Expansion (MoMA, 2020a), liberó cinco volúmenes de diferentes gases nobles inodoros e incoloros (helio, neón, argón, criptón y xenón) a la atmósfera. Se tomaron fotografías del acontecimiento, mismo que se llevó a cabo en Los Ángeles, y la única evidencia tangible que se conservó fue un póster publicado por el comerciante de arte neoyorkino Seth Siegelaub. En Ultrasonic Wave Piece (1968), Barry liberó ondas ultrasónicas en un espacio de exposición, las cuales eran inaudibles para los espectadores y generaban patrones. Con esto, intentaba cuestionar si una obra de arte existe con independencia de que pueda ser captada por los sentidos. 
Los trabajos de Rahm y Barry tienen en común la liberación de elementos imperceptibles en el espacio. En ambos casos podríamos apelar a la idea de 'conciencia' por la manera en que los creadores manipulan al espectador/usuario al modificar las acciones detrás de su entorno sin que él se dé cuenta hasta que es informado. Estas obras también nos remiten al concepto de "voluntad', porque el visitante se ve obligado, de alguna manera, a vivenciar un espacio que ha sido alterado para que se sienta más cómodo, en el caso de Rahm, o incómodo, en el trabajo de Barry (al retirar la posibilidad de que el espectador pueda advertir su trabajo).

Las propuestas que recién revisamos difieren en sus objetivos. Mientras que la del arquitecto apunta a volver más agradable un espacio público, la del artista plantea una reflexión sobre la desmaterialización del arte. Otro contraste que conviene subrayar es que Jade Eco Park e Inert Gas Series se centran en el espacio exterior, mientras que Ultrasonic Wave Piece ocurre en el interior, lo cual nos permite vislumbrar un panorama amplio en el que instalaciones y arquitectura tienen como materia de creación áreas interiores, exteriores o los límites que existen entre ellas.

\section{Caso 3: el uso de elementos portátiles no inflables}

El espacio portátil, particularmente las casas móviles, ha despertado el interés del mundo del arte y la arquitectura. Abordaremos este tema a partir de un número de la obra editorial Roulotte, de los españoles Xavier Arenós (1968), Domènec (1962) y Martí Peran, que recopila el trabajo de un grupo heterogéneo de artistas, fotógrafos, arquitectos e investigadores; y de una instalación del artista austriaco Erwin Wurn (1954).

Roulotte forma parte de una iniciativa mayor denominada Post-it City, un proyecto de investigación y archivo cuyo objetivo es analizar las maneras en que los espacios urbanos residuales son activados temporalmente por uno o más grupos humanos a la vez. Según Giovanni La Varra, quien acuñó el término en 2001:
Como un texto lleno de post-it, la ciudad con- temporánea está ocupada temporalmente por comportamientos que no dejan rastro - como tampoco los post-it en los libros-, que apa- recen y desaparecen de modo recurrente, que tienen sus formas de comunicación y de atrac- ción pero que cada vez resultan más difíciles de ignorar (2008: 14).

Roulotte, que significa 'caravana' en francés, es el nombre de la revista editada por Arenós, Domènec y Peran, quienes la han descrito como una exposición móvil que recopila información sobre proyectos de diferente índole creados para contextos particulares. En total, se publicaron once números de Roulotte, el primero de ellos en mayo de 2006 y el último en julio de 2015. Destaca el cuarto número, que salió a la luz en septiembre de 2007, dedicado por completo a las casas rodantes y caravanas desde la mirada de distintos artistas, fotógrafos, arquitectos e investigadores. Roulotte: 04 se estructuró en torno a dos ejes, darle preferencia al área metropolitana de Barcelona y registrar los diferentes modelos y usos de las casas rodantes.

En el ámbito de las artes visuales, Erwin Wurn inició su serie One Minute Sculpture en 1988. A partir de entonces ha estado interesado en señalar la función común de los objetos cotidianos y en alterar su uso establecido (Zilkha 2016). Wurn explica su trabajo como 'performative sculptures' [esculturas performativas] (MM-ArtLike, 2018), porque se necesita que el espectador use su cuerpo para terminarlas, siguiendo las instrucciones escritas o ilustradas por el artista, con lo cual pasa de ser un observador pasivo a ser observado por los demás. En su obra Just about Virtues and Vices in General (2016-2017) presenta, entre otras cosas, una casa rodante para explorar la idea de lo inmóvil y lo móvil, centrándose en la manera en que estos vehículos pueden 
reemplazar a las viviendas estáticas, pero también para generar una reflexión sobre la migración. Esa caravana corresponde a un modelo alemán de los años sesenta que las personas procedentes de este país usaban para viajar a Italia en busca de una mejor calidad de vida, llevando consigo todas sus pertenencias.

Roulotte: 04 y Just about Virtues and Vices in General tienen en común su interés por la naturaleza móvil de la casa rodante y también su intención de reflexionar sobre la migración que se da usando este tipo de transporte. Sin embargo, se distinguen en que Roulotte: 04 recopila muchos modelos de caravanas para tener una mejor comprensión de este dispositivo, sus materiales, su manera de modificar el espacio urbano residual y de generar relaciones humanas, mientras que Just about Virtues and Vices in General presenta un único modelo para repensar, entre otras cosas, las muchas historias de migración que se dieron en cierto momento histórico.

Estos trabajos también difieren en el formato en el que se crearon. El primero constituye un proyecto de investigación cercano al ámbito urbanístico que ha sido impreso. El segundo nace como una instalación concebida para presentarse en un espacio expositivo. Este contraste conduce a diferentes formas de interacción ya señaladas por los creadores de cada obra. Si consideramos Roulotte: 04 una exposición móvil, notaremos que es ella la que se mueve entre los espectadores, mientras que si tomamos a Just about Virtues and Vices in General como un conjunto de esculturas performativas, distinguiremos que son los observadores quienes deben desplazarse entre los elementos de la instalación fija.

\section{Caso 4: el uso de elementos portátiles inflables}

Nuestro último caso de estudio se refiere a un par de obras, una arquitectónica y una instalación, que comparten el uso de elementos portátiles inflables, recurso que ha estado presente en la historia de ambas disciplinas. En arquitectura, encontramos el Centro de refugiados, del alemán Hans-Walter Müller (1935), creado en 2016 en Lyon, Francia. Este sitio fue concebido para recibir en un espacio solidario a los inmigrantes que llegan a la ciudad. El edificio está integrado por dos zonas, la recepción, que se encuentra bajo una cúpula inflable, y el área de refugios. La sección inflable se forma por tres esferas que convergen entre sí formando un trébol y ocupa una superficie de $900 \mathrm{~m}^{2}$. Cada esfera corresponde a una zona diferente, una para menores de edad, mujeres y familias; otra para hombres solteros; y una más que funciona como sala de espera. Para crear este edificio y otros, Müller dibujó plantillas y las cortó en PVC, para después unirlas y finalmente inflar la estructura.

Desde las artes visuales, paraSITE homeless shelter fue creada en 1997 por el estadounidense Michael Rakowitz (1973). Esta pieza consiste en un conjunto de refugios individuales hechos con materiales como bolsas de plástico de cierre, cinta adhesiva y láminas de polietileno. Su principal característica, y de la cual deriva el nombre de este proyecto, es que, como un parásito, cada refugio se conecta a un ducto de calefacción de un edificio, gracias a lo cual se infla y mantiene caliente a quien esté en su interior. El proceso de diseño inició con una entrevista entre el artista y el ocupante, y culminó con un refugio hecho de acuerdo con sus necesidades. Algunos usuarios pidieron que tuviera cierta forma o ventanas para ver desde el interior si había alguna amenaza afuera. El objetivo de paraSITE homeless shelter fue "ofrecer algo de alivio a las personas que viven en las calles y agitar para que se realicen cambios políticos que resolverían permanentemente el problema de la falta de vivienda" (MoMA, (2020b). ${ }^{4}$

El Centro de refugiados de Lyon y paraSITE homeless shelter comparten el uso de elementos inflables, pero además son parecidos en cuanto

4 "offer some relief to people living in the streets and to agitate for policy changes that would permanently solve the problem of homelessness" [La traducción es mía]. 
a su espíritu humanitario y en la preocupación por ofrecer refugio a quien lo necesita, ya sea por su condición de inmigrantes o por estar en situación de calle. Se trata de dos trabajos con objetivos diferentes. La obra arquitectónica ofrece una solución, de manera puntual, a la necesidad de evitar la multiplicación de zonas sin condiciones dignas en la ciudad; mientras que la instalación fue creada para pedir la generación de políticas públicas que resuelvan las necesidades de las personas en situación de calle. Otro rasgo que comparten estas propuestas es que ambas benefician a un grupo de individuos y, sin embargo, lo hacen desde espacios totalmente diferentes. El Centro de refugiados opera desde la colectividad, aunque distinguiendo entre grupos de personas de acuerdo con su edad, sexo o estado civil; mientras que paraSITE homeless shelter favorece a un colectivo, pero ofreciendo refugios individuales y personalizados.

\section{Conclusiones}

A partir del ejercicio realizado a lo largo de este artículo es posible encontrar las siguientes coincidencias al interior de cada caso de estudio: One-Sqm-House y Measurement Rooms son similares porque intentan modificar el pensamiento del espectador/usuario respecto al espacio y revelan las dimensiones de los elementos que lo integran; Jade Eco Park y las instalaciones Inert Gas Series y Ultrasonic Wave Piece tienen en común la liberación de elementos imperceptibles; Roulotte: 04 y Just about Virtues and Vices in General se parecen en su interés por la casa rodante y su intención de reflexionar sobre la migración apoyada en ese dispositivo; el Centro de refugiados de Lyon y paraSITE homeless shelter comparten el uso de elementos inflables y su búsqueda por beneficiar a un grupo de personas.

Por otra parte, el análisis nos permite localizar las siguientes divergencias: One-Sqm-House y Measurement Rooms son diferentes en que la obra arquitectónica (que usa letras para indicar las dimensiones del espacio) persigue que el usuario entienda el espacio exterior como una extensión del interior, mientras que la instalación (que emplea números para revelar el tamaño del lugar) reflexiona sobre la importancia de la idea que genera un espacio respecto al espacio mismo; Jade Eco Park y las instalaciones Inert Gas Series y Ultrasonic Wave Piece difieren en que las primeras fueron hechas con el objetivo de volver más cómodo un espacio público exterior, mientras que la última fue concebida para el interior y reflexiona sobre la desmaterialización del arte; Roulotte: 04 y Just about Virtues and Vices in General, con distintos formatos y formas de interacción con el espectador, también se distinguen entre sí en que la primera recopila documentación de muchos modelos de casas rodantes para tener una mejor comprensión de un solo dispositivo, y la segunda, presenta un ejemplar de caravana para repensar muchas historias de migración; el Centro de refugiados de Lyon y paraSITE homeless shelter contrastan en que la obra arquitectónica ofrece una solución a un problema social y opera desde la colectividad, mientras que la instalación es más bien una petición y brinda refugios individuales y personalizados.

Este estudio nos lleva también a descubrir otros vínculos interesantes entre ambas disciplinas, más allá de los casos de estudio presentados. Aunque siempre podemos encontrar alguna similitud, hay proyectos relacionados con la arquitectura e instalaciones que difieren tanto en los recursos visuales empleados como en sus objetivos, como podemos descubrir si comparamos Jade Eco Park, de Philippe Rahm, y Measurement Rooms, de Mel Bochner. Por otra parte, el presente texto nos conduce a admitir que, aunque siempre descubriremos alguna diferencia, en ciertos casos los elementos usados y propósitos son muy parecidos, por ejemplo, en el caso del Centro de refugiados, de Hans-Walter Müller, y paraSITE homeless shelter, de Michael Rakowitz. 
También hemos encontrado que hay propuestas arquitectónicas e instalaciones que comparten recursos visuales y difieren en su intención, por ejemplo, Jade Eco Park, de Philippe Rahm e Inert Gas Series y Ultrasonic Wave Piece, de Robert Barry. En contraste, algunas tienen los mismos intereses, pero emplean distintos elementos, como Roulotte: 04, de Arenós, Domènec y Peran; y Just about Virtues and Vices in General, de Erwin Wurn.

Finalmente, si bien la selección de obras que se hizo para elaborar este artículo se centró en los recursos visuales que han sido compartidos por la arquitectura y las instalaciones, es importante subrayar que este estudio comparativo nos permite notar que existen muchos otros aspectos que aproximan o alejan entre sí a estas dos disciplinas (como los objetivos, la metodología o la interacción con el usuario/espectador) en un amplio universo por explorar.

\section{REFERENCIAS}

Centro Pompidou Málaga (2018), «Construir el aire. Arquitectura y diseño hinchable, 1960-1975», Málaga, Centro Pompidou, disponible en: https:/centrepompidou-malaga. eu/exposicion/construir-el-aire-arquitectura-y-diseno-hinchable-1960-1975/

CNN (2012), "Why make the 'world's smallest house?", disponible en: https://youtu.be/1CmrbQNWQwg

Dempsey, Amy (2010), Styles, Schools and Movements: The Essential Encyclopaedic Guide to Modern Art, Londres, Thames and Hudson.

La Varra, Giovanni (2008), "El último espacio público de la ciudad contemporánea”, en Giovanni La Varra, Martí Peran, Robert Kronenburg et al., Post-it City, Barcelona, Centre de Cultura Contemporània de Barcelona.

Larrañaga, Josu (2006), Instalaciones, Donostia-San Sebastián, Nerea.

MM-ArtLike (2018), "Austria - Erwin Wurm - Just About Virtues and Vices in General - Venice Biennale 2017”, disponible en: https://www.youtube.com/watch?v=ekzXOtdyVi8

Museum of Modern Art (MoMA) (2020), "Robert Barry. Inert Gas Series/Helium, Neon, Argon, Krypton, Xenon/From a Measured Volume to Indefinite Expansion, 1969”, en "Art and artist", disponible en: https://www.moma.org/collection/works/1097100020
Museum of Modern Art (MoMA) (2020), paraSITE homeless shelter, en MoMA Learning, disponible en: https:/www. moma.org/learn/moma_learning/michael-rakowitz-parasite-homeless-shelter-1997)/

Rahm, Philippe (2016), Jade Eco Park, en About Philippe Rahm, disponible en: http://www.philipperahm.com/data/ projects/taiwan/index.html

Zilkha, Zmira (2016), "Erwin Wurm, One Minute Sculptures, 1997", en "Art \& artists. TATE", disponible en: https:qq9//www.tate.org.uk/art/artworks/wurm-one-minute-sculptures-p82013
Gonzalo Enrique Bernal Rivas. Doctor en Artes Visuales e Intermedia por la Universidad Politécnica de Valencia (España). Está adscrito a la Universidad de Guanajuato, donde se orienta académicamente por la arquitectura efímera, las instalaciones y la transdisciplina. Es también colaborador de la revista .925 Artes y Diseño de la Facultad de Artes y Diseño de la Universidad Nacional Autónoma de México (UNAM), México, plantel Taxco, y de la revista Radar del Centro. 


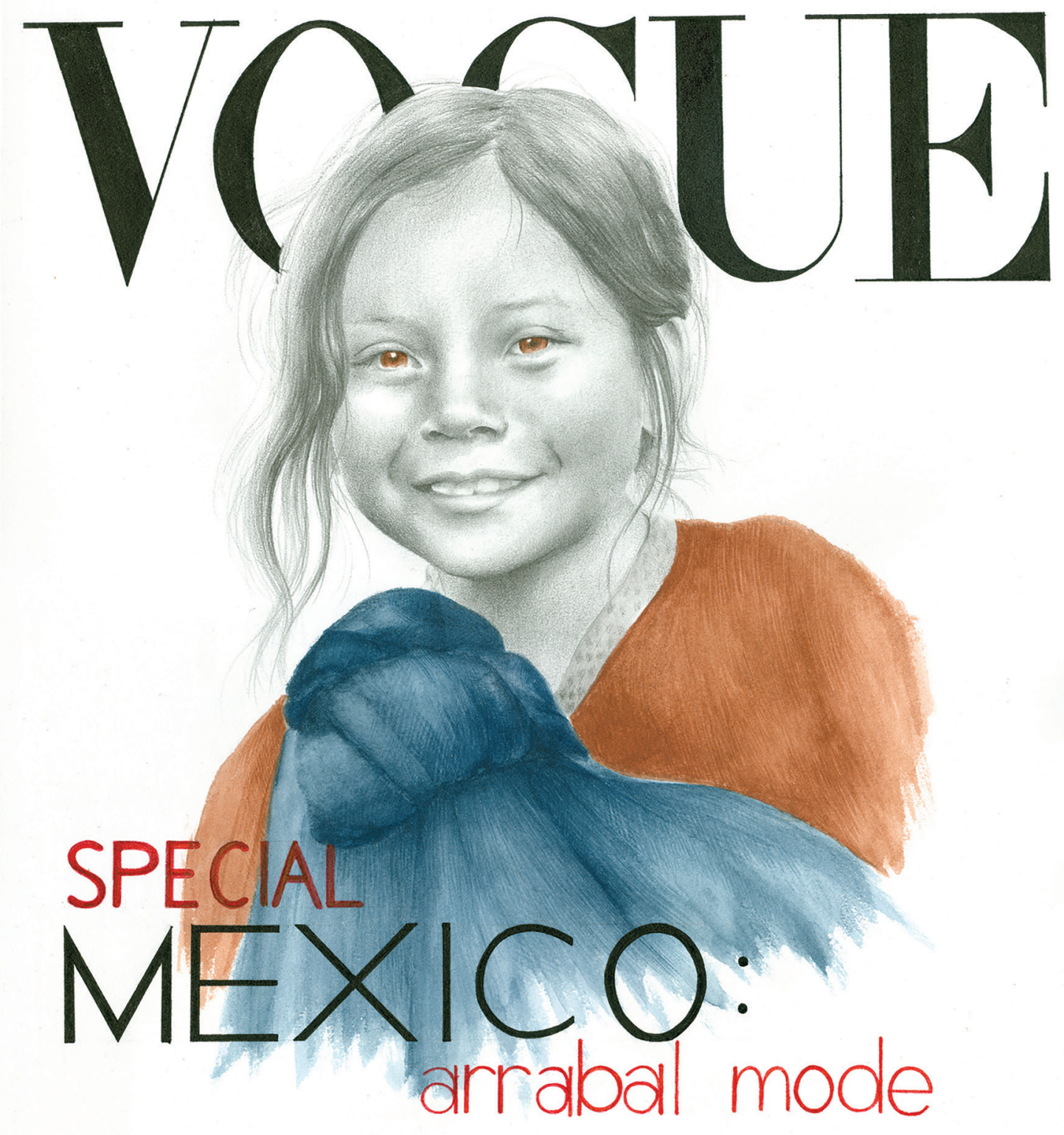

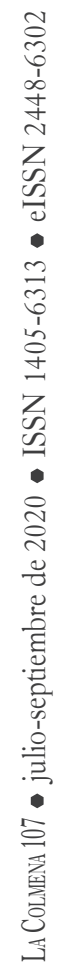

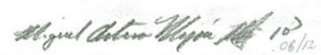

Mexican Vogue de la serie Entremés (2010). Grafito y acuarela sobre papel:

Miguel Arturo Mejía-Martínez.

Prohibida su reproducción en obras derivadas. 